\title{
Model and Topological Characteristics of Power Distribution System Security Region
}

\author{
Jun Xiao, Guo-qiang Zu, Xiao-xu Gong, and Cheng-shan Wang \\ Key Laboratory of Smart Grid of Ministry of Education, Tianjin University, Nankai District, Tianjin 300072, China \\ Correspondence should be addressed to Jun Xiao; xiaojun@tju.edu.cn and Guo-qiang Zu; zuguoqiang_tju@163.com
}

Received 16 January 2014; Accepted 28 June 2014; Published 24 July 2014

Academic Editor: H. D. Chiang

Copyright (C) 2014 Jun Xiao et al. This is an open access article distributed under the Creative Commons Attribution License, which permits unrestricted use, distribution, and reproduction in any medium, provided the original work is properly cited.

\begin{abstract}
As an important tool of transmission system dispatching, the region-based method has just been introduced into distribution area with the ongoing smart distribution grid initiatives. First, a more accurate distribution system security region (DSSR) model is proposed. The proposed model is based on detailed feeder-interconnected topology, and both substation transformer and feeder $\mathrm{N}-1$ contingencies are considered. Second, generic characteristics of DSSR are discussed and mathematically proved. That is, DSSR is a dense set of which boundary has no suspension and can be expressed by several union subsurfaces. Finally, the results from both a test case and a practical case demonstrate the effectiveness of the proposed modeling approach; the shape of DSSR is also illustrated by means of 2- and 3-dimensional visualization. Moreover, the DSSR-based assessment and control are preliminary illustrated to show the application of DSSR. The researches in this paper are fundamental work to develop new security region theory for future distribution systems.
\end{abstract}

\section{Introduction}

This paper presents a model of power distribution system security region (DSSR) which takes both substation transformer and feeder N-1 contingencies into account. Meanwhile, main topological characteristics of DSSR are also discussed and mathematically proved.

DSSR is a newly proposed concept originated from N1 security guideline $[1,2]$. DSSR is defined as the set of all operating points that make the distribution system $\mathrm{N}-1$ secure, taking into account the capacities of substation transformers, network topology, network capacity, and operational constraints. Traditional method of security assessment and control focused on service restoration in which load transfer scheme is made on case by case N-1 test $[3,4]$. However, the "point-wise" simulation method only provides the binary information of security and insecurity, which causes global information and description of the security boundary to fail to be obtained [5]. Moreover, with the number of interconnected feeders growing in distribution systems, especially in urban areas, the simulation time of "point-wise" method that has to be calculated online is highly prohibitive. In contrast, the "region" method has advantages in dealing with the problems above.

In transmission system, the research on security region has made substantial achievements. Author of [6-8] described the topological characteristics for security region and defined the practical model for engineering application. However, the "region" method has scarcely been applied in distribution area. Because early systems were little more than an extension of SCADA beyond the substation fence, most operations of 10 or $20 \mathrm{kV}$ feeders required a high degree of human intervention [9]. Plus, without high speed peerto-peer communication system, a wealth of key operation information cannot be timely received by dispatchers, which limited the actual contribution of "region" method. Distribution automation (DA) is an important concept included in smart distribution grid and will upgrade the distribution systems to fully information-based systems $[10,11]$. That is a motivation for researchers to perform the research on "region" method aiming at distribution system.

References [12,13] proposed the concept of loadability. It is defined as the maximum loading level that can be supplied. Loadability is similar to the proposed "region" 


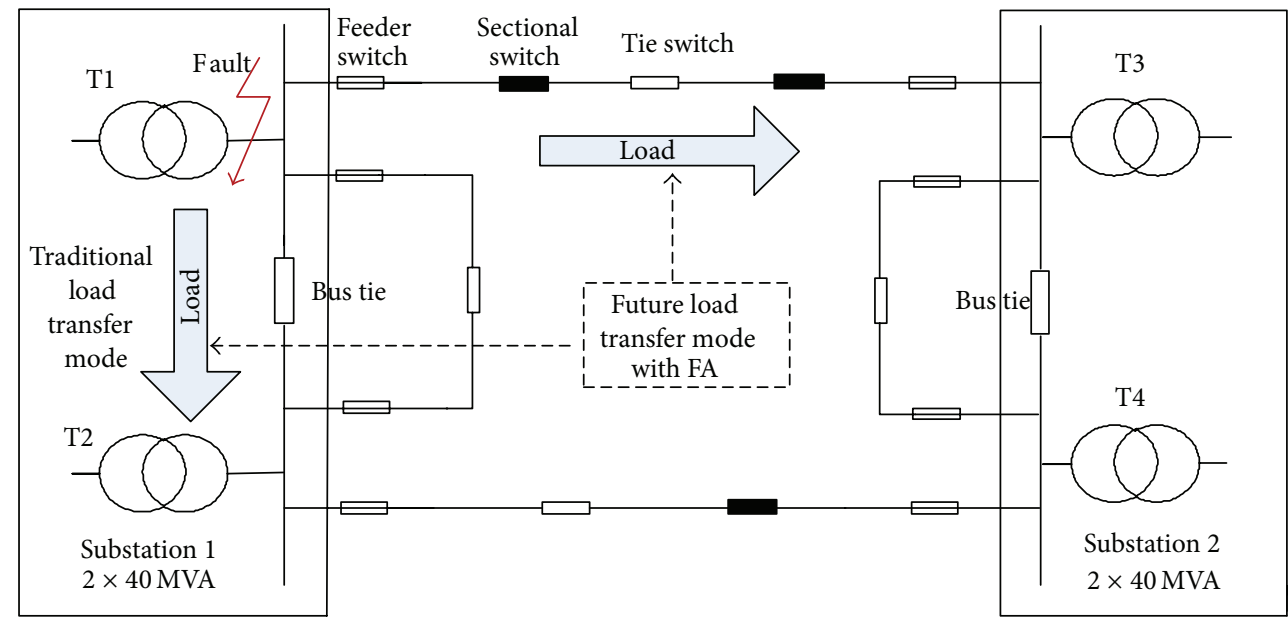

FIGURE 1: Load transfer mode.

concept. However, because of not considering N-1 cases, the concept is not suitable for urban system where $\mathrm{N}-1$ security is a high concern.

Total supply capability (TSC) is a newly proposed concept which is defined as the capability that a system can supply the load when the N-1 security for distribution systems is considered $[14,15]$. At the TSC point, the facilities in the system are usually fully utilized. Although some TSC results can be used as the points of DSSR boundary [15], TSC fails to describe the integrated boundary. Moreover, TSC cannot obtain the relative location of an operating point in security region, which is very useful for dispatchers to make decisions. In a word, TSC is more suitable to be applied in planning than operating process.

Author of [1] made attempt to present a simplified model and approach of security assessment with the concept of DSSR. But the papers exposed 2 main defects: (1) only the load of substation transformers and their rated capacities are formulated, which provide relatively limited information on the system status; (2) feeder contingency and maintenance are ignored although feeder contingency occurs much more frequently than transformer contingency.

The DSSR theory has potential applications. However, some wrong conclusions will be reached if the topological characteristics of DSSR are not studied. Paper [16] designed an N-1 approximating approach to study the characteristics of DSSR. But those characteristics have not been proved by rigorous mathematical method and some of them are inaccurately described.

This paper proposes a more accurate DSSR model. The mathematical proof for the characteristics of DSSR is also performed. Finally, a test case and a practical case are both selected to testify the proposed model.

\section{Basic Concept and Method}

2.1. N-1 Security for Distribution System. The DSSR concept is based on contingency scenarios. Feeder contingency and substation transformer contingency are two main scenarios considered in security analysis. Here, if a distribution system is feeder N-1 secure, it means that when a fault occurs at outlet of any main feeder, the corresponding load can be transferred to other feeders with a series of tie-switch operations. Meanwhile, all components in the system operate normally without overload or overvoltage. Similarly, if a distribution is substation transformer N-1 secure, it means that when a fault occurs at any substation transformer, the corresponding load can be transferred to other transformers with all the components satisfying the operation constraints. The load of a transformer can be transferred in two ways: one way is to be transferred to the adjacent transformers in the same substation with bus-tie switches; the other way is to be transferred to other substations by tie-switch operations among feeders.

2.2. Load Transfer and Concept of DSSR. Feeders in traditional medium-voltage network are not completely automated. When a fault occurs at a substation transformer, load will be only transferred to adjacent transformers in the same substation, even though feeders form connection between substations. Manual performing for complex circuitrestoration switching takes time and is not performed in practical operation. Thus, it causes a lower load ratio of substation transformers, which also means a conservative utilization of assets.

However, development of smart distribution grid has greatly promoted the level of feeder automation, especially in some core urban area. Distributed intelligence and automated switching accomplish the task, that is, to transfer load among different substations quickly, accurately, and flexibly. In other words, switches that the planner formerly could not tie can now be tied, resulting in higher load ratio within security constraints. This draws the attention to a more accurate calculation of security boundary. Figure 1 shows the load transfer mode after feeder automation (FA).

As is shown in Figure 1, when a fault occurs at T1, in traditional way, all of the load of $\mathrm{T} 1$ will be only transferred to T2, because the time of load transfer through feeders is 


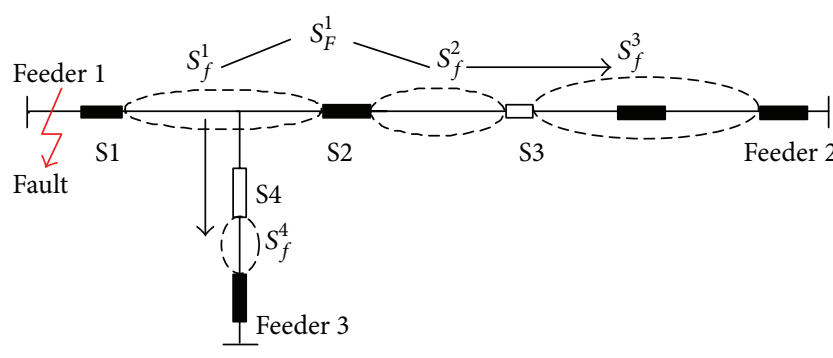

\footnotetext{
- Normally closed switch

$\neg$ - Normally open switch

$\longrightarrow$ Back-feed (transfer) path
}

Figure 2: Feeder and transfer unit load.

too long. In contrast, with feeder automation, the load of T2 can be transferred not only to $\mathrm{T} 1$, but also to $\mathrm{T} 3$ in another substation.

The DSSR model in this paper takes both substation transformer N-1 and feeder N-1 contingency into account. The DSSR is defined as the set of operating points which assure the N-1 security of distribution system. An operating point in transmission system is the current power injections of nodes, while, in distribution system, it represents a set of transfer unit load. Consider

$$
\mathbf{W}=\left(S_{f}^{1}, S_{f}^{2}, \ldots, S_{f}^{n}\right)^{T},
$$

whereW is the operating point, which is an $n$-dimension vector; $S_{f}^{i}$ is transfer unit load. "Transfer unit" is defined as the set of feeder sections of which load has the same backfeed (transfer) path. If two feeders form single loop network, transfer unit load is equivalent to feeder load. If a feeder is connected to two other feeders, which is called two-tie connection, the feeder load can be divided into two parts of load, each of which is a transfer unit load. In this paper, feeder load $i$ is denoted by $S_{F}^{i}$ and transfer unitload $i$ is denoted by $S_{f}^{i}$. Take the brief case in Figure 2 as an example.

In Figure 2, feeder 1 forms two-tie connection and feeder 2 and feeder 3 form single loop network, respectively, with feeder 1. In normal state, $S_{f}^{1}$ and $S_{f}^{2}$ are both supplied by feeder 1; that is, $S_{F}^{1}=S_{f}^{1}+S_{f}^{2}$. When a fault occurs at outlet of feeder 1, S1 and S2 disconnect and S4 closes, and then $S_{f}^{1}$ changes to be supplied by feeder 3 , while $S_{f}^{2}$ is supplied by feeder 2 via closing S3. Transfer scheme is usually not unique. Another scheme is that transferring both $S_{f}^{1}$ and $S_{f}^{2}$ to feeder 2 by disconnecting $\mathrm{S} 1$ and closing S3. However, multischeme makes the DSSR model overcomplicated. Thus, scheme is fixed in this paper, which stipulates that each of the backup feeders restores only one section of the whole faulted feeder, as the first scheme above. More important, this fixed scheme can usually balance the branch load in the postfault network, which is an important index concerned by dispatchers.

The DSSR model in this paper is accurate to the safety monitoring of transfer unit load. We can certainly get feeder load by summing up the transfer unit load and further get transformer load by summing up the feeder load. This is a basis for DSSR model considering both transformer N-1 and feeder N-1 security.

\section{Mathematical Model for DSSR}

After the load transfer incurred by tie switches against N1 contingency, all the transformers and feeders cannot be overloading. Since the tie-line is designed after the radial network planning, the capacity is considered as enough to transfer load. Therefore, the transformer load and feeder load should be under a series of constraints. The DSSR model can be mathematically formulated as

$$
\Omega_{\mathrm{DSSR}}=\{\mathbf{W} \mid h(x) \leq 0 \quad g(x)=0\},
$$

where $\mathbf{W}=\left(S_{f}^{1}, S_{f}^{2}, \ldots, S_{f}^{n}\right)^{T}$ is the operating point corresponding to $S_{f}^{1}, S_{f}^{2}, \ldots, S_{f}^{n}$. The inequality and equality constraints are such that

$$
\begin{gathered}
S_{F}^{m}=\sum_{n=1} S_{f, t r}^{m, n}, \\
S_{f, t r}^{m, n}+S_{F}^{n} \leq S_{F, \max }^{n} \quad(\forall m, n), \\
S_{T, t r}^{i, j}=\sum_{m \in \Phi^{(i)}, n \in \Phi^{(j)}} S_{f, t r}^{m, n}, \\
S_{T}^{i}=\sum_{m \in \Phi^{(i)}} S_{F}^{m} \quad(\forall i), \\
S_{T, t r}^{i, j}+S_{T}^{j} \leq S_{T, \max }^{j},
\end{gathered}
$$

where $S_{F}^{m}=$ all the load supplied by feeder $m ; S_{f, t r}^{m, n}=$ the load transferred from feeder $m$ to feeder $n$ when an $\mathrm{N}-1$ fault occurs at outlet of feeder $m ; S_{F, \max }^{n}=$ maximal thermal capacity of feeder $n ; S_{T, t r}^{i, j}=$ the load transferred from transformer $i$ to transformer $j$ when an $\mathrm{N}-1$ fault occurs at transformer $i ; \Phi^{(i)}=$ the set of feeders that derive from transformer $i ; S_{T}^{j}=$ all of the load supplied by transformer $i$; $m \in \Phi^{(i)}$ means that feeder $m$ derives from the corresponding bus of transformer $i ; S_{T, \max }^{i}=$ the rated capacity of transformer $i$.

It is notable that the correspondence relation of " $S_{f, t r}$ " and " $S_{f}$ " depends on the load transfer scheme. For example, in Figure 2, in the former scheme, $S_{f, t r}^{1,2}=S_{f}^{2}$; in the latter scheme, $S_{f, t r}^{1,2}=S_{f}^{1}+S_{f}^{2}$.

Equation (3) shows that the sum of each load to be transferred away from feeder $m$ should be equal to the total load supplied by feeder $m$ in normal state; inequality constraint (4) means that feeders cannot be overloading in the postfault network; (5) shows that path of load transferred from transformer $i$ to $j$ is the tie-lines between transformers $i$ and $j$. Equation (6) means that load of transformer is equal to the sum of corresponding feeder loads; inequality constraint (7) describes that the load of transformer $j$ cannot exceed its rated capacity after a substation transformer $\mathrm{N}-1$ contingency.

It should be pointed out that power flow and voltage drop are not taken into consideration in this DSSR model, which 
determines that the DSSR model is completely linear. Since overloading under contingencies is the most critical problem in urban power, the simple linear model is acceptable from the standpoint of security-based operation [1]. More importantly, research of [16] has demonstrated that the DSSR model is approximately linear even when considering factors of power flow and voltage drop, which further proves that the linear model is highly approximate to the real DSSR model.

\section{Formulation for DSSR Boundary}

Research on the boundary of security region has vital significance, because the characteristics determine the application way of DSSR theory [7]. The proposed DSSR model, which is formulated as (2)-(7), cannot distinctly express the DSSR boundary. Therefore, derivation should be performed to transform the original model to a new expression form. Select $S_{f, t r}^{u, v}$ as research object, $u \in \Phi^{(i)}$ and $v \in \Phi^{(j)}$. First, formula (4) can be transformed to

$$
S_{f, t r}^{u, v} \leq S_{F, \max }^{v}-S_{F}^{v}
$$

Second, substitute formulas (5) and (6) into (7), and then perform identical deformation. We obtain

$$
0 \leq S_{T, \max }^{j}-\sum_{t \in \Phi^{(j)}} S_{F}^{t}-\sum_{m \in \Phi^{(i)}, n \in \Phi^{(j)}} S_{f, t r}^{m, n}
$$

Third, add $S_{f, t r}^{u, v}$ on both sides of formula (9), and then

$$
S_{f, t r}^{u, v} \leq S_{T, \max }^{j}-\sum_{t \in \Phi^{(j)}} S_{F}^{t}-\sum_{m \in \Phi^{(i)}, n \in \Phi^{(j)}, m \neq u, n \neq v} S_{f, t r}^{m, n} .
$$

Finally, according to (8) and (10), the expression of DSSR boundary in direction of $S_{f, t r}^{u, v}$ can be neatly formulated as

$$
\begin{aligned}
& S_{f, t r}^{u, v} \\
& \leq \min \left\{S_{F, \max }^{v}-S_{F}^{v}, S_{T, \max }^{j}-\sum_{t \in \Phi^{(j)}} S_{F}^{t}-\sum_{\substack{m \in \Phi^{(i)}, n \in \Phi^{(j)} \\
m \neq u, n \neq v}} S_{f, t r}^{m, n}\right\} .
\end{aligned}
$$

There are $n$ boundaries if the system consists of $n$ load transfer units. To express the dimension of boundary clearly, we denote all " $S_{f, t r}^{u, v}$ " by $S_{f, t r}^{1}, S_{f, t r}^{2}, \ldots, S_{f, t r}^{n}$. Because the transfer scheme in this paper is fixed, as is illustrated in Section 2.2, an important equivalence holds as follows:

$$
S_{f, t r}^{i}=S_{f}^{i} \quad(i=1,2, \ldots, n) .
$$

Through (12), $S_{f, t r}$ and $S_{f}$ have one-to-one corresponding relation. Above all, the complete and succinct boundary formulation for DSSR is expressed as

$$
\begin{aligned}
& \Omega_{\text {DSSR }} \\
& =\left\{\begin{array}{l}
B_{1} \\
B_{2} \\
\vdots \\
B_{i} \\
\vdots \\
B_{n}
\end{array}\right. \\
& =\left\{\begin{array}{l}
S_{f}^{1} \leq \min \left\{S_{F, \max }^{b 1}-S_{F}^{b 1}, S_{T, \max }^{b 1}-\sum_{j \in \Phi^{(b 1)}} S_{F}^{j}-\sum_{k \neq 1, k \in \Theta^{(1)}} S_{f}^{k}\right\} \\
S_{f}^{2} \leq \min \left\{S_{F, \max }^{b 2}-S_{F}^{b 2}, S_{T, \max }^{b 2}-\sum_{j \in \Phi^{(b 2)}} S_{F}^{j}-\sum_{k \neq 2, k \in \Theta^{(2)}} S_{f}^{k}\right\} \\
\vdots \\
S_{f}^{i} \leq \min \left\{S_{F, \max }^{b i}-S_{F}^{b i}, S_{T, \max }^{b i}-\sum_{j \in \Phi^{(b i)}} S_{F}^{j}-\sum_{k \neq i, k \in \Theta^{(i)}} S_{f}^{k}\right\} \\
S_{f}^{n} \leq \min \left\{S_{F, \max }^{b n}-S_{F}^{b n}, S_{T, \max }^{b n}-\sum_{j \in \Phi^{(b n)}} S_{F}^{j}-\sum_{k \neq n, k \in \Theta^{(n)}} S_{f}^{k}\right.
\end{array}\right\}
\end{aligned}
$$

With the form transformation of DSSR model, corresponding symbols and their meaning should be adjusted too. Thus, $b i$ and $\Theta^{(i)}$ are used for new expression. $b i=$ the number of back-feed feeders of $S_{f}^{i}$; $S_{F}^{b i}=$ all of the load supplied by the back-feed feeder $b i$; $\Theta^{(i)}=$ the set of transfer unit loads, of which both normal-feed and back-feed transformer are the same as those of $S_{f}^{i}$. It should be pointed out that feeder $b 1 \ldots$ feeder $b n$ are not $n$ different actual feeders. Take the case in Figure 2 as an example again; when fault occurs, the backfeed feeders of $S_{f}^{3}$ and $S_{f}^{4}$ are both feeder 1, which means feeder $b 3=$ feeder $b 4=$ feeder 1 . When any equality in (13) holds, the operating point is just upon the DSSR boundary. We denote the $n$ boundaries by $B_{1}, \ldots, B_{n}$.

The DSSR boundary formulation can also reflect both substation transformer and feeder N-1 secure constraints. Take $i$ subformula of (13) to concretely explain; $S_{f}^{i} \leq$ $S_{F, \max }^{b i}-S_{F}^{b i}$ shows that transfer of $S_{f}^{i}$ cannot cause the feeder overloading, which ensures that the feeder N-1 is secure; $S_{f}^{i} \leq$ $S_{T, \max }^{b i}-\sum_{j \in \Phi^{(b i)}} S_{F}^{j}-\sum_{k \neq 1, k \in \Theta^{(i)}} S_{f}^{k}$ shows that transfer of $S_{f}^{i}$ cannot lead to the substation transformer overloading, which ensures that the transformer $\mathrm{N}-1$ is secure.

\section{Topological Characteristics of DSSR and Mathematical Proof}

The research on differential topological characteristics of the DSSR focuses on several aspects as follows. 
(1) Are there any holes inside the DSSR, that is, the denseness in terms of the topology?

(2) Does the boundary of the DSSR have suspension?

(3) Could the boundary of the DSSR be expressed with the union of several subsurfaces?

The identical deformation from formulas (2)-(7) to (13) is a breakthrough for the research on topological characteristics of the DSSR. However, to research on these characteristics, formula (13) should be further simplified to obtain the mathematical essence. Take $i$ subformula of (13) as research object; the equivalent form is

$$
\begin{gathered}
S_{f}^{i}+S_{F}^{b i} \leq S_{F, \max }^{b i}, \\
S_{f}^{i}+\sum_{j \in \Phi^{(b i)}} S_{F}^{j}+\sum_{k \neq 1, k \in \Theta^{(i)}} S_{f}^{k} \leq S_{T, \max }^{b i} .
\end{gathered}
$$

According to formulas (3) and (12), $S_{F}^{b i}$ and $S_{F}^{j}$ can be expressed as linear combination of a series of different element " $S_{f}$." Then, formula (14) will be simplified as

$$
\begin{aligned}
& \sum_{m=1}^{n} \alpha_{m} S_{f}^{m} \leq S_{F, \text { max }}^{b i}, \\
& \sum_{m=1}^{n} \beta_{m} S_{f}^{m} \leq S_{T, \text { max }}^{b i},
\end{aligned}
$$

where $S_{F, \max }^{b i}$ and $S_{T, \max }^{b i}$ are positive constants. Through formula (14), coefficients $\alpha$ and $\beta$ have the following features:

$$
\begin{aligned}
& \alpha_{m}=1, \quad \beta_{m}=1 \quad(m=i), \\
& \alpha_{m}=0 \text { or } 1, \quad \beta_{m}=0 \text { or } 1 \quad(m \neq i) .
\end{aligned}
$$

Let $\Omega_{\mathrm{DSSR}}$ be a set which consists of operating point $\mathbf{W}$. DSSR boundary formulation can be abstracted as

$$
\mathrm{AW} \leq \mathrm{C},
$$

where $\mathbf{W}$ is a vector defined as formula (1). Through formulas (15)-(16), matrices A and C should satisfy

$$
\begin{aligned}
\mathbf{A} & =\left[a_{i j}\right]_{2 n \times n}, \quad i \geq 1, j \geq 1, \\
a_{i j} & =1 \quad(i=2 j-1 \text { or } i=2 j), \\
a_{i j} & =0 \text { or } 1, \quad(i \neq 2 j-1, i \neq 2 j), \\
\mathbf{C} & =\left[c_{1}, c_{2}, \ldots c_{2 n}\right]^{T}, \quad \mathbf{C} \in \mathbf{R}^{+} .
\end{aligned}
$$

Because one integrated subformula is divided into equivalent two parts, as is shown in formula (15), the dimension of A is $2 n \times n$ instead of $n \times n$. Here, $\Omega_{\mathrm{DSSR}}$ is both linear space and Euclidean space, which has been proved in [17]. Besides this important premise, we should review some preparation definitions and theorems about topology and Euclidean space, including cluster point, closure, dense set, and hyperplane.
Definition 1 (cluster point). Let $\mathbf{x}$ be a point in Euclidean space, $F \subset R$. If there is a point sequence in set $F$ converging to $\mathbf{x}$, then $\mathbf{x}$ is the cluster point of set $F$ [17].

Definition 2 (closure). $A$ is a subset of topological space; $\bar{A}$ represents all the points inside $A$ and cluster points of $A$, and then $\bar{A}$ is called closure of $A[18]$.

Theorem 3. If the $B$ is a subset of $A, \bar{B}=A$, then $B$ is dense in A [18].

Theorem 4. Let $L$ be a subspace of linear space $R ; \mathbf{x}_{0}$ is a fixed vector which does not belong to L generally. Considering set $H$ which consists of the vector $\mathbf{x}, \mathbf{x}$ is obtained by

$$
\mathbf{x}=\mathbf{x}_{\mathbf{0}}+\mathbf{y}
$$

where vector $\mathbf{y}$ varies in the whole subspace L. Thus, $H$ is called hyperplane. The dimension of $H$ equals that of subspace L [17].

The topological characteristics of DSSR are mathematically proved in the following section.

Characteristic 1. The $\Omega_{\mathrm{DSSR}}$ is dense inside.

Proof. First, a new set $\Omega_{\mathrm{DSSR}}^{\prime}$ is defined as the set of all points of $\Omega_{\mathrm{DSSR}}$ except those on boundaries. $\Omega_{\mathrm{DSSR}}^{\prime}$ meets

$$
\mathrm{AW}<\mathrm{C} \text {. }
$$

Select randomly a vector $\mathbf{W}=\left(S_{f}^{1}, S_{f}^{2}, \ldots, S_{f}^{n}\right)^{T}$ from $\Omega_{\mathrm{DSSR}}^{\prime}$, and then construct a sequence $\mathbf{Y}=\left\{\mathbf{Y}_{1}, \mathbf{Y}_{2}, \ldots, \mathbf{Y}_{\mathbf{m}}, \ldots\right\}$ as the following formula:

$$
\mathbf{Y}=\left\{\begin{array}{rl}
\mathbf{Y}_{1} & =\left(S_{f}^{1}-\frac{S_{f}^{1}}{1}, S_{f}^{2}-\frac{S_{f}^{2}}{1}, \ldots, S_{f}^{n}-\frac{S_{f}^{n}}{1}\right)^{T} \\
& =(0,0, \ldots, 0)^{T} \\
\mathbf{Y}_{2} & =\left(S_{f}^{1}-\frac{S_{f}^{1}}{2}, S_{f}^{2}-\frac{S_{f}^{2}}{2}, \ldots, S_{f}^{n}-\frac{S_{f}^{n}}{2}\right)^{T} \\
\vdots & \\
\mathbf{Y}_{m} & =\left(S_{f}^{1}-\frac{S_{f}^{1}}{m}, S_{f}^{2}-\frac{S_{f}^{2}}{m}, \ldots, S_{f}^{n}-\frac{S_{f}^{n}}{m}\right)^{T} \\
& =\left(1-\frac{1}{m}\right) \mathbf{W}
\end{array} .\right.
$$

According to (20)-(21), we obtain

$$
\mathbf{A Y}_{\mathbf{m}}=\mathbf{A}\left(1-\frac{1}{m}\right) \mathbf{W}<\mathbf{A W}<\mathbf{C}
$$

Therefore, sequence $\mathbf{Y}=\left\{\mathbf{Y}_{1}, \mathbf{Y}_{2}, \ldots, \mathbf{Y}_{\mathbf{m}}, \ldots\right\}$ is inside $\Omega_{\mathrm{DSSR}}^{\prime}$. Let $\rho\left(\mathbf{W}, \mathbf{Y}_{\mathbf{m}}\right)$ be the distance between $\mathbf{W}$ and $\mathbf{Y}_{\mathbf{m}}$ in Euclidean space, and then

$$
\lim _{m \rightarrow \infty} \rho\left(\mathbf{W}, \mathbf{Y}_{\mathbf{m}}\right)=\lim _{m \rightarrow \infty} \sqrt{\sum_{i=1}^{n}\left[S_{f}^{i}-\left(S_{f}^{i}-\frac{S_{f}^{i}}{m}\right)\right]^{2}}=0 .
$$



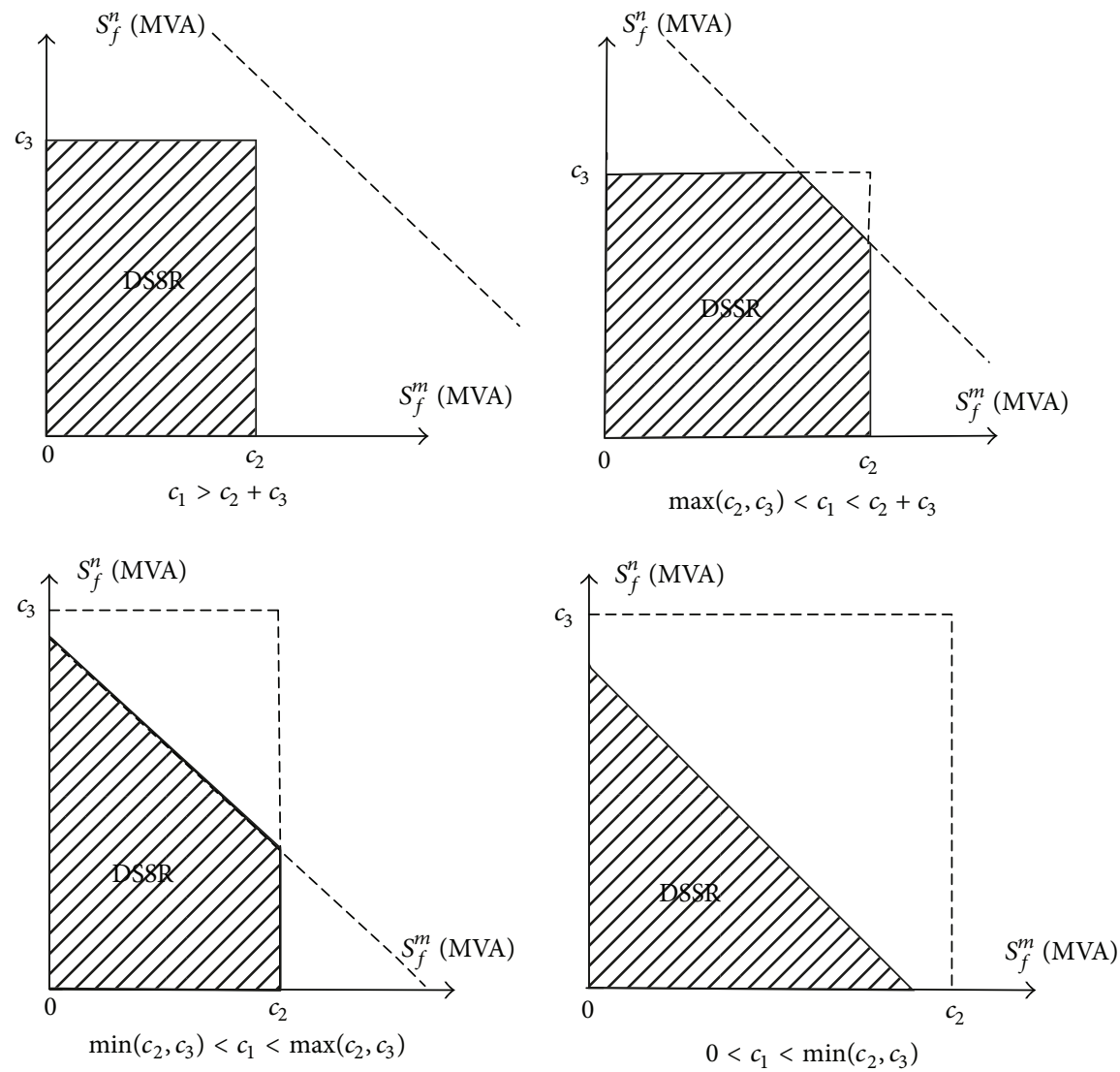

FIGURE 3: Shapes of 2-dimension cross-section of DSSR.

This means that, for any vector $\mathbf{W}$ in $\Omega_{\text {DSSR }}^{\prime}$, sequence $\mathbf{Y}$ exists and converges to $\mathbf{W}$, and $\mathbf{Y}$ is also inside $\Omega_{\mathrm{DSSR}}^{\prime}$. Therefore, any point inside $\Omega_{\text {DSSR }}^{\prime}$ is cluster point of $\Omega_{\text {DSSR }}^{\prime}$.

Similarly, select vector $\mathbf{Z}=\left(S_{f}^{1}, S_{f}^{2}, \ldots, S_{f}^{n}\right)^{T}$ which is just upon boundary of $\Omega_{D S S R}$ :

$$
\mathbf{A Z}=\mathbf{C} .
$$

Construct a sequence $\mathbf{U}=\left\{\mathbf{U}_{1}, \mathbf{U}_{2}, \ldots, \mathbf{U}_{\mathbf{m}}, \ldots\right\}$ :

$$
\mathbf{U}_{\mathbf{m}}=\left(1-\frac{1}{m}\right) \mathbf{Z}
$$

Then we obtain

$$
\mathbf{A U}_{\mathbf{m}}=\mathbf{A}\left(1-\frac{1}{m}\right) \mathbf{Z}<\mathbf{A Z}=\mathbf{C} .
$$

According to formula (26), sequence $\mathbf{U}$ is also inside $\Omega_{\mathrm{DSSR}}^{\prime}$. Through the following

$$
\lim _{m \rightarrow \infty} \rho\left(\mathbf{Z}, \mathbf{Y}_{\mathbf{m}}\right)=\lim _{m \rightarrow \infty} \sqrt{\sum_{i=1}^{n}\left[S_{f}^{i}-\left(S_{f}^{i}-\frac{S_{f}^{i}}{m}\right)\right]^{2}}=0 .
$$

We obtain that any point just upon the $\Omega_{\text {DSSR }}$ boundary is also cluster point of $\Omega_{\mathrm{DSSR}}^{\prime}$. Above all, cluster points of $\Omega_{\mathrm{DSSR}}^{\prime}$ include not only all the points inside $\Omega_{\text {DSSR }}^{\prime}$ but also all the points just upon the boundaries of $\Omega_{\text {DSSR }}$. Thus, the following formula holds:

$$
\overline{\Omega_{\mathrm{DSSR}}^{\prime}}=\Omega_{\mathrm{DSSR}}
$$

Through Theorem 3, $\Omega_{\mathrm{DSSR}}^{\prime}$ is dense in $\Omega_{\mathrm{DSSR}}$. This proof is completed.

Characteristic 2. The boundary of $\Omega_{\mathrm{DSSR}}$ has no suspension.

Proof. This characteristic can be approximately simplified to prove that any 2-dimension cross-section of $\Omega_{\mathrm{DSSR}}$ is convex polygon. For $\mathbf{W}=\left(S_{f}^{1}, S_{f}^{2}, \ldots, S_{f}^{n}\right)^{T}$, select a 2 -dimension cross-section by taking $S_{f}^{m}$ and $S_{f}^{n}$ as variables and $S_{f}$ in another dimension as constants. According to formula (16), the coefficient of $S_{f}$ (including variable $S_{f}$ and constant ones) can be only 1 or 0 ; thus, in process of dimension reduction, $S_{f}^{m}$ and $S_{f}^{n}$ are simply constrained by

$$
\begin{gathered}
S_{f}^{m}+S_{f}^{n} \leq c_{1}, \\
0 \leq S_{f}^{m} \leq c_{2}, \\
0 \leq S_{f}^{n} \leq c_{3}, \\
\forall c_{1}, c_{2}, c_{3} \in R^{+} .
\end{gathered}
$$


TABle 1: Profile of test case.

\begin{tabular}{lccccc}
\hline Substation & Transformer & Voltages $(\mathrm{kV} / \mathrm{kV})$ & Transformer capacity (MVA) & Number of feeders & Total feeder capacity (MVA) \\
\hline \multirow{2}{*}{ S1 } & T1 & $35 / 10$ & 40.0 & 9 & 80.28 \\
& T2 & $35 / 10$ & 40.0 & 9 & 80.28 \\
\hline \multirow{2}{*}{ S2 } & T3 & $35 / 10$ & 40.0 & 9 & 80.28 \\
& T4 & $35 / 10$ & 40.0 & 10 & 71.36 \\
S3 & T5 & $110 / 10$ & 63.0 & 10 & 89.2 \\
& T6 & $110 / 10$ & 63.0 & 10 & 89.2 \\
\multirow{2}{*}{ S4 } & T7 & $110 / 10$ & 63.0 & 10 & 89.2 \\
& T8 & $110 / 10$ & 63.0 & & 89.2 \\
\hline
\end{tabular}

The shape of 2-dimension cross-section varies with the values of $c_{1}, c_{2}$, and $c_{3}$, as is shown in Figure 3. The dashed oblique line represents $S_{f}^{m}+S_{f}^{n} \leq c_{1}$.

As is shown in Figure 3, the shapes of 2-dimension section are confined to rectangle, triangle, ladder, and convex pentagon. This means that any 2-dimension section of DSSR is convex polygon, which proves that the boundary of $\Omega_{\mathrm{DSSR}}$ has no suspension in an indirect way. The proof is completed.

Characteristic 3. The $\Omega_{\text {DSSR }}$ boundary can be expressed with several subsurfaces.

Proof. $H$ is a set of vector $\mathbf{W}=\left(S_{f}^{1}, S_{f}^{2}, \ldots, S_{f}^{n}\right)^{T}$, each of which satisfies nonhomogeneous linear equation set

$$
\mathrm{AW}=\mathrm{C},
$$

where matrices $\mathbf{A}$ and $\mathbf{C}$ meet formula (18) and $\mathbf{W}$ is the operating point just upon the boundary of $\Omega_{\mathrm{DSSR}}$. Let $\mathbf{W}_{\mathbf{0}}=$ $\left(\theta_{1}^{(0)}, \theta_{2}^{(0)}, \ldots, \theta_{n}^{(0)}\right)^{T}$ be a fixed solution of equation set $(31)$. Meanwhile, $L$ is a subspace of $R$, which consists of $\mathbf{y}=$ $\left(\lambda_{1}, \lambda_{2}, \ldots, \lambda_{n}\right)^{T}$; that is, $\mathbf{y}$ varies in the whole subspace $L$. The coordinates of $\mathbf{y}$ meet the following homogeneous linear equation set:

$$
\text { Ay }=0 \text {. }
$$

If $\mathbf{y}=\left(\lambda_{1}, \lambda_{2}, \ldots, \lambda_{n}\right)^{T}$ is a solution of (31), $\mathbf{W}=\mathbf{W}_{\mathbf{0}}$ $+\mathbf{y}=\left(\theta_{1}^{(0)}+\lambda_{1}, \theta_{2}^{(0)}+\lambda_{2}, \ldots, \theta_{n}^{(0)}+\lambda_{n}\right)^{T}$ is obviously a solution of equation set (30); that is, $\mathbf{W}$ is included in set $H$. Meanwhile, $\mathbf{x}_{\mathbf{0}}$ is not in $L$. Through Theorem 3, H is hyperplane. The boundary of $\Omega_{\mathrm{DSSR}}$ can be expressed with hyperplane, that is, several subsurfaces. Because the dimension of $L$ is $n$, the dimension of hyperplane is also $n$. This proof is completed.

In transmission area, topological characteristics of security region (SR) have been deeply studied [7]. It has shown that the boundary of SR has no suspension and is compact and approximately linear; there is no hole inside the SR. The proof above demonstrates that characteristics of DSSR are similar but it has its own features. (a) DSSR is a convex set of denseness in terms of the topology. This means that DSSR has no holes inside and its boundary has no suspension. Based on this feature, dispatchers can analyze the security of operating point by judging whether the point is inside the DSSR boundary, without being afraid of any insecure point inside or losing security points.

(b) DSSR boundary can be described by hyperplane (several Euclidean subsurfaces); meanwhile, the boundary is more linear than that of SR. In practical application, calculation of high dimensional security region will incur great computation burdens and consume large amount of time. Therefore, this characteristic will improve the computational efficiency.

\section{Case Study}

In order to verify the effectiveness of the proposed model, two cases are illustrated in this section. The first one is a test case, and the second is a practical case.

\subsection{Test Case}

6.1.1. Overview of Test Case. As is shown in Figure 4, the grid is comprised of 4 substations, 8 substation transformers, and 75 feeders. 68 feeders form 34 single loop networks, and 7 form two-tie connections, resulting in 82 load transfer units. The total capacity of substations is $412 \mathrm{MVA}$. To illustrate the modeling process clearly, each load transfer unit is numbered. Case profile is shown in Table 1.

The conductor type of all feeders is LGJ-185 (8.92 MVA). This type is selected based on the national electrical code in China.

6.1.2. Boundary Formulation. The test case grid has 75 feeders; 14 feeders form 7 two-tie connections. Each load of these 7 feeders can be divided into 2 parts to transfer in different back-feed path. So the number of load transfer units is 82 , which means that the dimension of operation point $\mathbf{W}$ is 82 and the number of subformulas of DSSR boundary is 82. Based on the topology of the test case, we substitute the rated capacity of substation transformers and feeders into $S_{F, \max }^{1}, \ldots, S_{F, \max }^{n}$ and $S_{T, \text { max }}^{1}, \ldots, S_{T, \max }^{n}$ of formula (13). 
Then, the boundary formulation for DSSR of test case can be expressed as

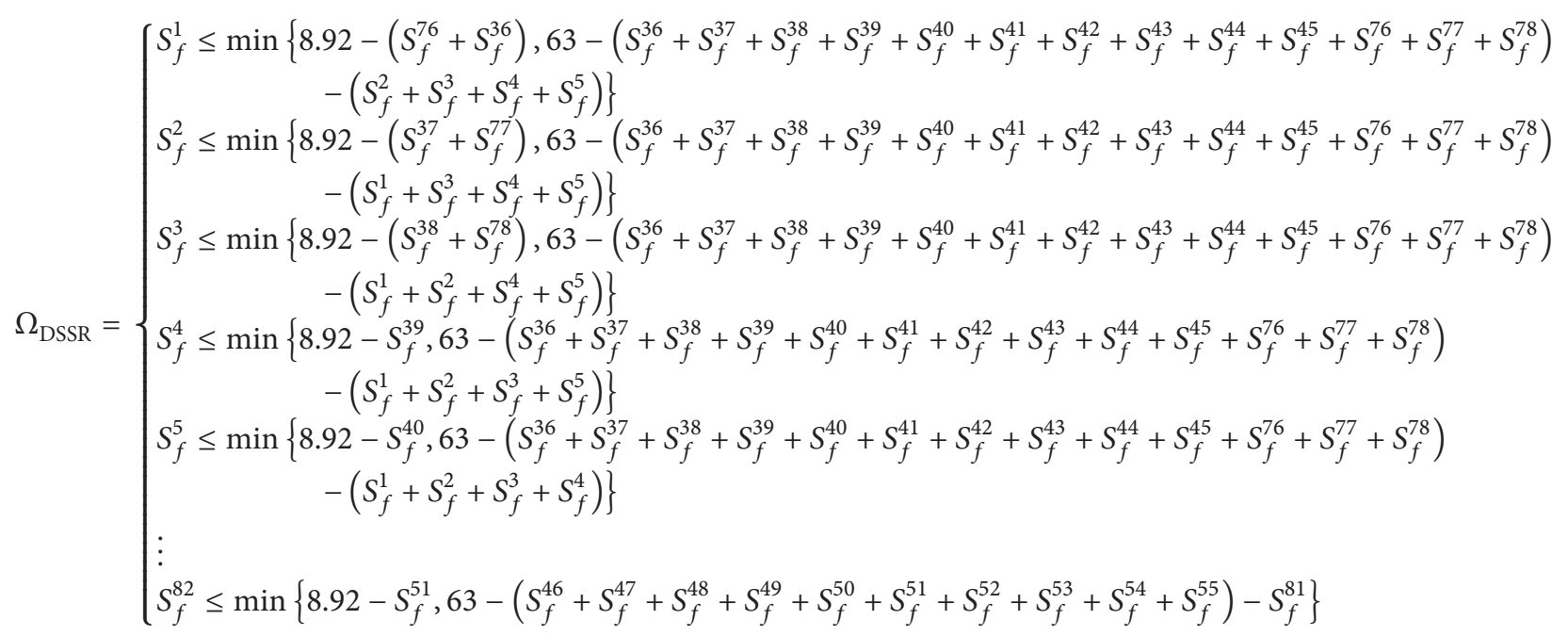

Considering the similarity of calculation approach of each boundary and thesis length, we present 5 subformulas of $S_{f}^{1}, \ldots, S_{f}^{5}$ here. Complete formulation is given in the appendix (in the Supplementary Material available online at http://dx.doi.org/10.1155/2014/327078). Take the first subformula in (32) as an example to explain the modeling process. Figure 5 shows the local topology related with $S_{f}^{1}$, including all relative transfer units in the first subformula.

First, analyze the topology and determine the parameters of formula (13). We know that back-feed feeder of $S_{f}^{1}$ is feeder 36 and the back-feed substation transformer of $S_{f}^{1}$ is T5. In normal state, $S_{f}^{36}, \ldots, S_{f}^{45}$ and $S_{f}^{76}, \ldots, S_{f}^{78}$ are all load of T5, which means $\sum_{j \in \Phi^{(b 1)}} S_{F}^{j}=\sum_{j=36}^{45} S_{f}^{j}+\sum_{j=76}^{78} S_{f}^{j} ; S_{f}^{36}$ and $S_{f}^{76}$ are load of feeder 36 , which means $S_{F}^{b 1}=S_{f}^{36}+S_{f}^{76} \cdot S_{f}^{2}, \ldots, S_{f}^{5}$ are the load transferred from T1 to T5 when fault occurs at T1, which means $\sum_{k \neq 1, k \in \Theta^{(1)}} S_{f}^{k}=\sum_{k=2}^{5} S_{f}^{k}$. Therefore, the formula of $B_{1}$ is preliminary expressed as

$$
\begin{aligned}
S_{f}^{1} \leq \min \left\{S_{F, \max }^{36}-\right. & \left(S_{f}^{76}+S_{f}^{36}\right), \\
S_{T, \max }^{5}- & \left(S_{f}^{36}+S_{f}^{37}+S_{f}^{38}+S_{f}^{39}+S_{f}^{40}+S_{f}^{41} S_{f}^{42}\right. \\
& \left.+S_{f}^{43}+S_{f}^{44}+S_{f}^{45}+S_{f}^{76}+S_{f}^{77}+S_{f}^{78}\right) \\
-\left(S_{f}^{2}+\right. & \left.\left.S_{f}^{3}+S_{f}^{4}+S_{f}^{5}\right)\right\} .
\end{aligned}
$$

Second, according to case profile, substitute $S_{F, \max }^{36}=$ 8.92 MVA and $S_{T, \max }^{5}=63 \mathrm{MVA}$, into formula (33), and then we obtain

$$
\begin{aligned}
S_{f}^{1} \leq \min \{ & 8.92-\left(S_{f}^{76}+S_{f}^{36}\right), \\
& 63-\left(S_{f}^{36}+S_{f}^{37}+S_{f}^{38}+S_{f}^{39}+S_{f}^{40}+S_{f}^{41} S_{f}^{42}\right.
\end{aligned}
$$

$$
\begin{aligned}
& \left.+S_{f}^{43}+S_{f}^{44}+S_{f}^{45}+S_{f}^{76}+S_{f}^{77}+S_{f}^{78}\right) \\
& \left.-\left(S_{f}^{2}+S_{f}^{3}+S_{f}^{4}+S_{f}^{5}\right)\right\} .
\end{aligned}
$$

6.1.3. Visualization and Topological Characteristics. To visualize the shape of DSSR, the dimension is reduced to 2 and 3 , which means that the coordinates of $\mathbf{W}$ should be constants other than those to be visualized. Thus, an operating point has to be predetermined to provide the constants. TSC is a special operating point just upon the security boundary [1], on which the facilities are usually fully utilized. The operation of distribution system is closing to the boundaries and load level is reaching TSC. Thus, in this test case, we select the TSC point as the predetermined operating point $\mathbf{W}_{\mathrm{TSC}}$, which is shown in Table 2. The calculation approach for TSC has been presented in [15].

Choose randomly transfer unit loads $S_{f}^{15}$ and $S_{f}^{52}$ to observe. Substitute the rated capacity of transformers T1 T6, capacity of all feeders, and all transfer unit loads other than $S_{f}^{15}$ and $S_{f}^{52}$, into formula (32). Then the 2D DSSR shown in Figure 6 is obtained.

Although the dimension is 2 , the number of subformulas which constrain the figure shape is not just 2 . In fact, each of the subformulas which contains $S_{f}^{15}$ or $S_{f}^{52}$ should be considered. But the subformulas with more severe constraints will cover the others and finally form the DSSR boundary. The final formulation of $2 \mathrm{D}$ figure is

$$
\begin{gathered}
0 \leq S_{f}^{15} \leq 5.2, \\
0 \leq S_{f}^{52} \leq 3 .
\end{gathered}
$$




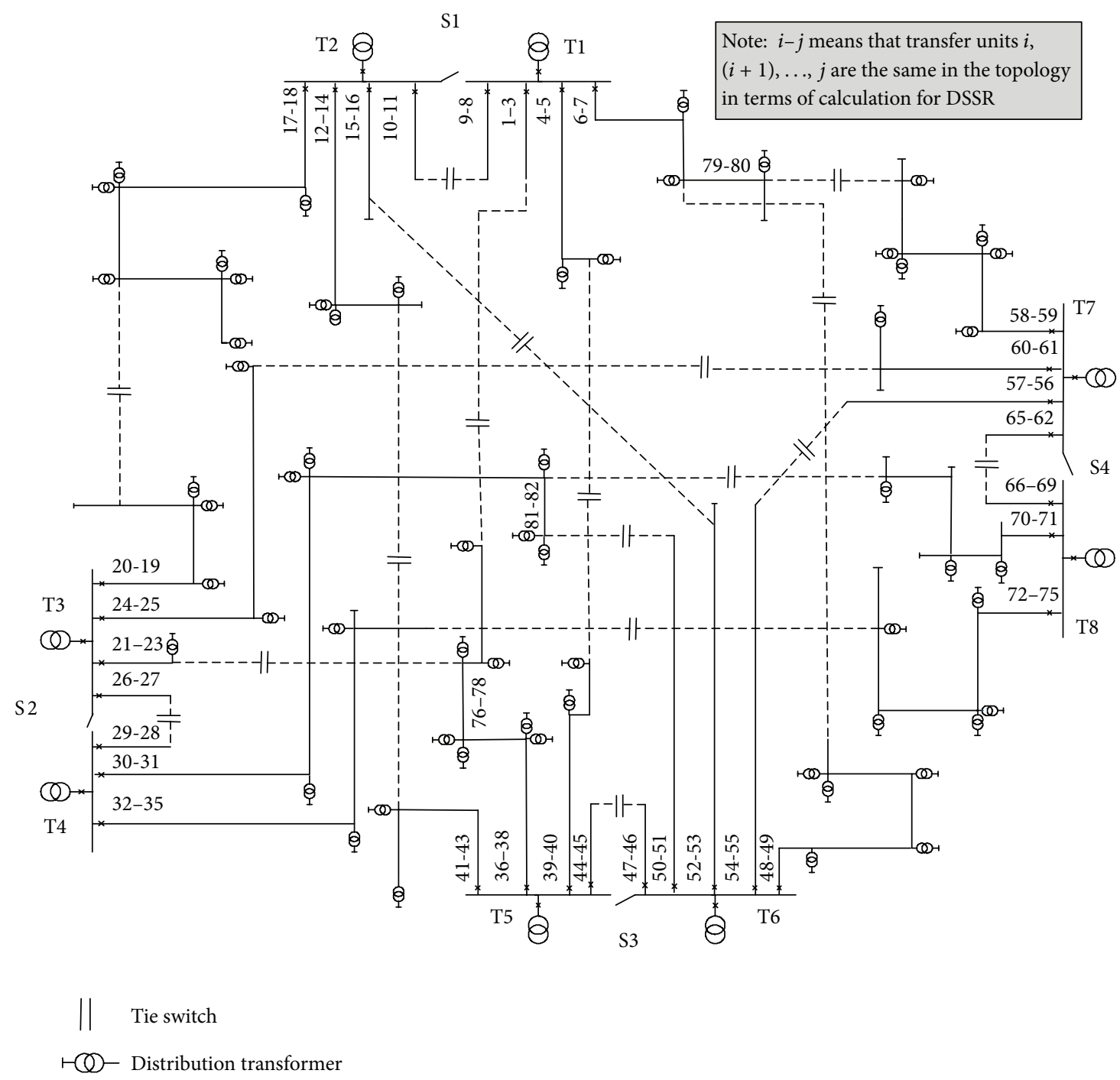

FIGURE 4: Test case with eight substation transformers.

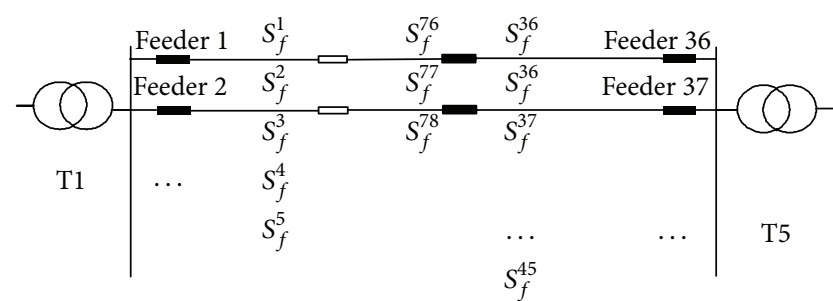

Figure 5: Local topology related with $f_{1}$.

Similarly, we choose randomly transfer unit loads $S_{f}^{19}, S_{f}^{24}$, and $S_{f}^{32}$ to observe, and then the 3D DSSR is obtained, as is shown in Figure 7.

In Figure 6, it can be seen that the 2D DSSR is a dense rectangle, of which boundaries are linear without suspension.

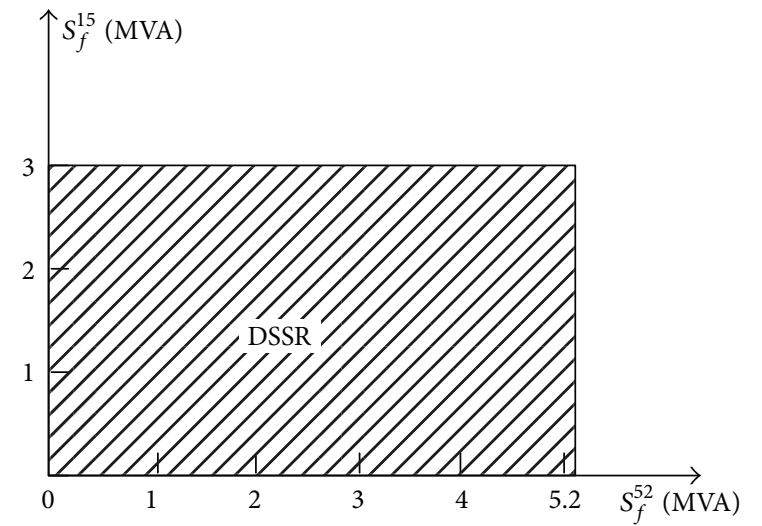

FIGURE 6: Shape of 2D DSSR.

In Figure 7, the 3D DSSR is surrounded by several subplanes, forming a convex pentagon, that is, a special 3-dimension hyperplane. 
TABLE 2: Transfer unit load of $\mathbf{W}_{\text {TSC }}$ in test case.

\begin{tabular}{|c|c|}
\hline Transfer unit & Load (MVA) \\
\hline 1 & 2.88 \\
\hline 2 & 2.88 \\
\hline 3 & 2.88 \\
\hline 4 & 2.88 \\
\hline 5 & 2.88 \\
\hline 6 & 1.64 \\
\hline 7 & 1.64 \\
\hline 8 & 1.64 \\
\hline 9 & 1.64 \\
\hline 10 & 2.88 \\
\hline 11 & 2.88 \\
\hline 12 & 3.17 \\
\hline 13 & 3.17 \\
\hline 14 & 3.17 \\
\hline 15 & 3.17 \\
\hline 16 & 3.17 \\
\hline 17 & 3.00 \\
\hline 18 & 3.00 \\
\hline 19 & 3.17 \\
\hline 20 & 3.17 \\
\hline 21 & 3.47 \\
\hline 22 & 3.47 \\
\hline 23 & 3.11 \\
\hline 24 & 3.11 \\
\hline 25 & 3.11 \\
\hline 26 & 3.47 \\
\hline 27 & 3.47 \\
\hline 28 & 3.47 \\
\hline 29 & 3.47 \\
\hline 30 & 2.80 \\
\hline 31 & 2.80 \\
\hline 32 & 1.61 \\
\hline 33 & 1.61 \\
\hline 34 & 1.61 \\
\hline 35 & 1.61 \\
\hline 36 & 2.80 \\
\hline 37 & 2.80 \\
\hline 38 & 2.80 \\
\hline 39 & 2.80 \\
\hline 40 & 2.52 \\
\hline 41 & 3.29 \\
\hline 42 & 2.52 \\
\hline 43 & 3.29 \\
\hline 44 & 2.52 \\
\hline 45 & 3.29 \\
\hline 46 & 2.88 \\
\hline 47 & 2.88 \\
\hline 48 & 3.95 \\
\hline 49 & 3.95 \\
\hline
\end{tabular}

TABle 2: Continued.

\begin{tabular}{|c|c|}
\hline Transfer unit & Load (MVA) \\
\hline 50 & 3.95 \\
\hline 51 & 4.46 \\
\hline 52 & 4.46 \\
\hline 53 & 4.46 \\
\hline 54 & 4.46 \\
\hline 55 & 5.64 \\
\hline 56 & 5.64 \\
\hline 57 & 5.69 \\
\hline 58 & 5.69 \\
\hline 59 & 5.92 \\
\hline 60 & 5.92 \\
\hline 61 & 5.33 \\
\hline 62 & 5.33 \\
\hline 63 & 3.59 \\
\hline 64 & 3.59 \\
\hline 65 & 5.13 \\
\hline 66 & 5.13 \\
\hline 67 & 4.94 \\
\hline 68 & 4.94 \\
\hline 69 & 4.46 \\
\hline 70 & 4.46 \\
\hline 71 & 4.46 \\
\hline 72 & 4.46 \\
\hline 73 & 4.46 \\
\hline 74 & 4.46 \\
\hline 75 & 4.46 \\
\hline 76 & 4.46 \\
\hline 77 & 2.80 \\
\hline 78 & 2.80 \\
\hline 79 & 2.80 \\
\hline 80 & 2.80 \\
\hline 81 & 2.80 \\
\hline 82 & 2.80 \\
\hline- & - \\
\hline - & - \\
\hline
\end{tabular}

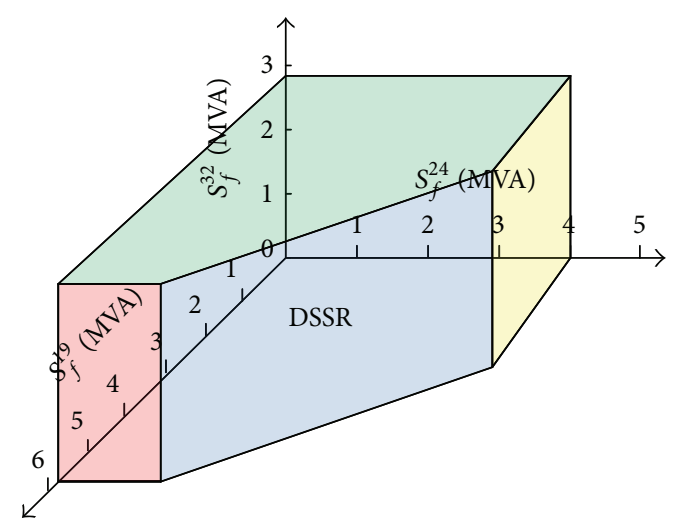

Figure 7: Shape of 3D DSSR. 


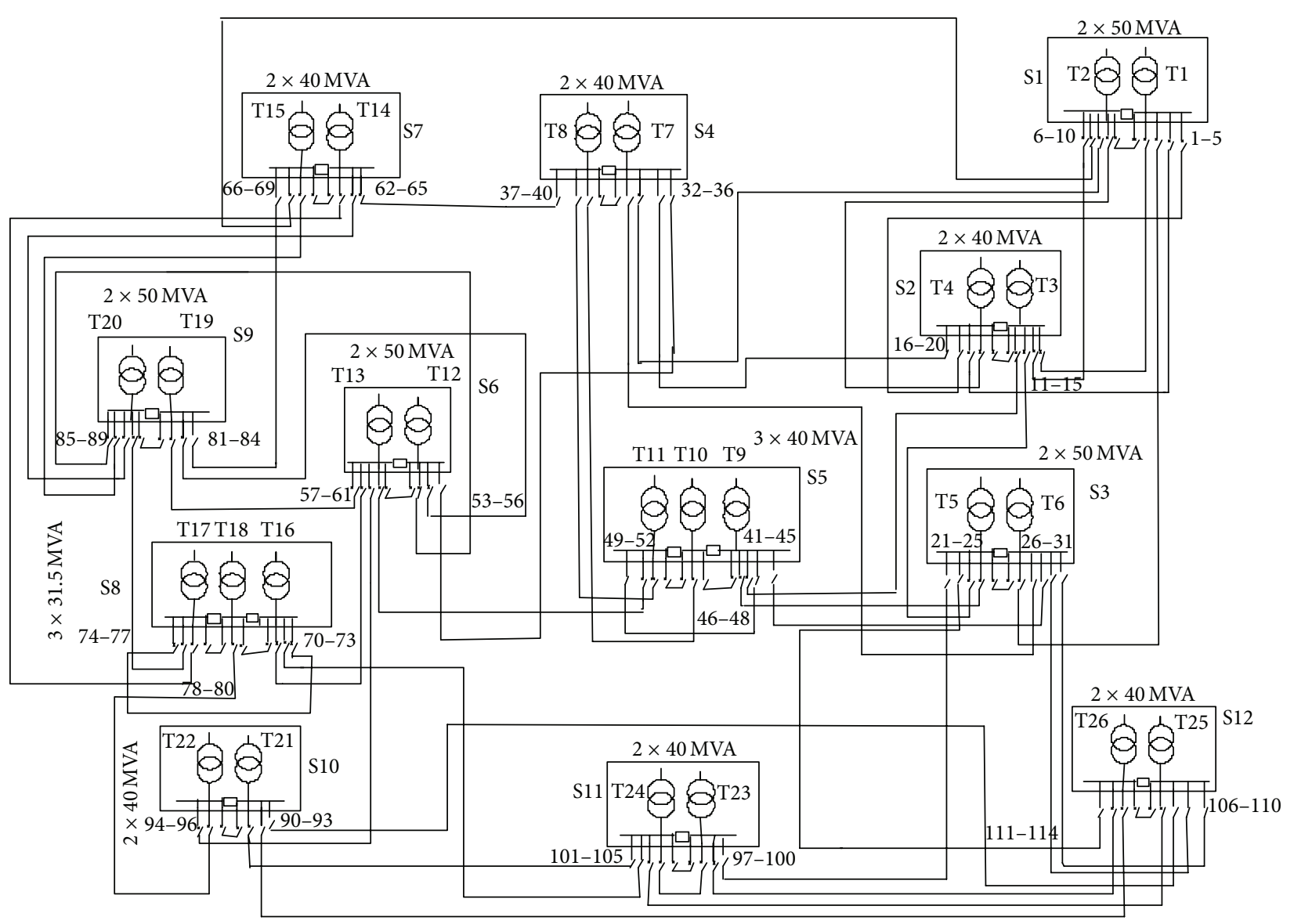

FIGURE 8: The medium-voltage urban power grid of one city of southern China.

6.2. Practical Case. In this section, the assessment and control method based on DSSR is demonstrated on a real medium-voltage distribution network of one city in southern China, which consists of 12 substations, 26 substation transformers, and 114 main feeders, as is shown in Figure 8. Total capacity of substation transformers is 1094.5 MVA. It is notable that each two of all main feeders form a single loop network in this case, which leads to the fact that the number of main feeders is equal to that of load transfer units.

Paper [1] has presented concept and calculation method of relative location of operating point in DSSR based on substation transformer contingency. Similarly, in this paper, relative location $L_{i}$ describes the distance from the operating point $\mathbf{W}$ to the boundary $B_{i}(i=1,2, \ldots, n)$ :

$L_{i}=\min \left\{S_{F, \max }^{b i}-S_{F}^{b i}, S_{T, \max }^{b i}-\sum_{j \in \Phi^{(b i)}} S_{F}^{j}-\sum_{k \neq i, k \in \Theta^{(i)}} S_{f}^{k}\right\}-S_{f}^{i}$.

$L_{i}$ is an index which is applied in security assessment. Here is an example. Let $\mathbf{W}_{\mathbf{1}}$ be an insecure operating point. Each transfer unit load of $\mathbf{W}_{\mathbf{1}}$ is 0.8 times of TSC load in the practical case, except $S_{f}^{1}$ and $S_{f}^{10}$, while $S_{f}^{1}=8 \mathrm{MVA}$ and $S_{f}^{10}=7$ MVA. Total load of $\mathbf{W}_{\mathbf{1}}$ is 508.7 MVA, while TSC is 628.9 MVA. Average load rate is 0.46 . That means that current
TABle 3: Distance to boundaries of $B_{1}$ and $B_{10}$ at $\mathbf{W}_{\mathbf{1}}$.

\begin{tabular}{lcc}
\hline$B_{i}$ & $B_{1}$ & $B_{10}$ \\
\hline$L_{i}(\mathrm{MVA})$ & 0.778 & 0.778 \\
\hline
\end{tabular}

operating point can be controlled back to security region. Through formula (36), the location of a given operating point in DSSR can be calculated. We present the $B_{i}$ of which $L_{i}$ are negative in Table 3.

Table 3 shows that the system should lose $3.222 \mathrm{MVA}$ if fault occurs at feeder 1 or feeder 10 , so the system is locally overloading. The location of $\mathbf{W}_{1}$ can be easily observed in Figure 9. To meet operational constraints under N-1 contingency, $S_{f}^{1}$ and $S_{f}^{10}$ should be adjusted. For example, $\mathbf{W}_{\mathbf{1}}$ is adjusted to $\mathbf{W}_{\mathbf{1}}^{\prime}$ through the dashed arrow shown in Figure 9. The location of $\mathbf{W}_{\mathbf{1}}^{\prime}$ is shown in Table 4.

\section{Conclusion and Further Work}

This paper proposes a mathematical model for distribution system security region (DSSR); generic topological characteristics of DSSR are discussed and proved.

First, the operating point for a distribution system is defined as the set of transfer unit loads, which is a vector in the 


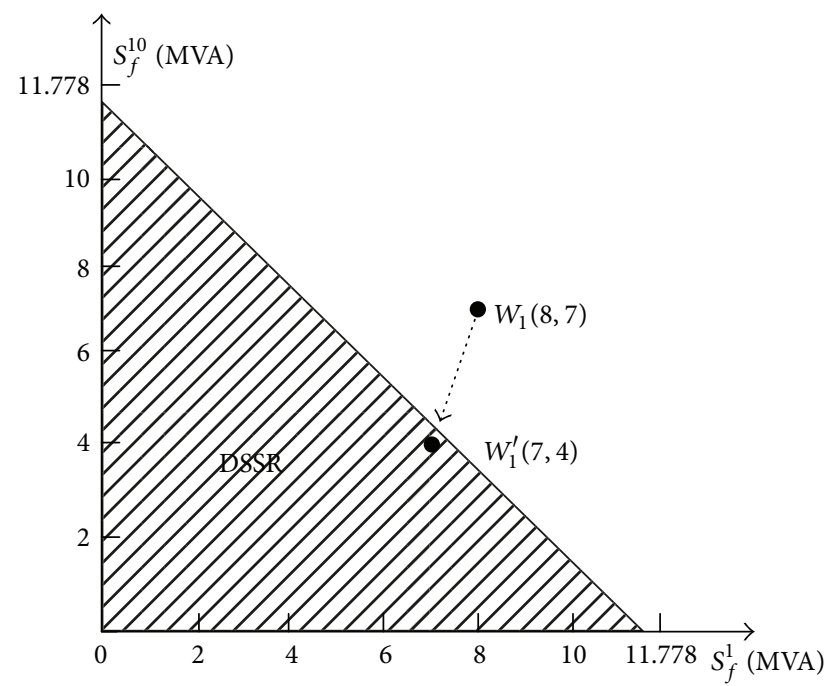

FIGURE 9: 2D DSSR cross-section and preventive control.

TABLE 4: Distance to boundaries of $B_{1}$ and $B_{10}$ at $\mathbf{W}_{\mathbf{1}}^{\prime}$.

\begin{tabular}{lcc}
\hline$B_{i}$ & $B_{1}$ & $B_{10}$ \\
\hline$L_{i}$ (MVA) & 0.778 & 0.778 \\
\hline
\end{tabular}

Euclidean space. Then, the DSSR model for both substation transformer and feeder contingency is proposed, which is the set of all operating points; each ensures that the system N-1 is secure.

Second, to perform research on the characteristic of DSSR, the boundary formulation for DSSR is derived from the DSSR model. Then, three generic characteristics are proposed and proved by rigor mathematical approach, which are as follows: (1) DSSR is dense inside; (2) DSSR boundary has no suspension; (3) DSSR boundary can be expressed with the union of several subsurfaces.

Finally, the results from a test case and a practical case demonstrate the effectiveness of the proposed model. The visualization for DSSR boundary is also discussed to verify the topological characteristics of DSSR. Security assessment method based on DSSR is preliminary exhibited to show the applicability of DSSR for future smart distribution system.

This paper improves the accuracy and understanding of DSSR, which is fundamental theoretic work for future smart distribution system. Further works to improve the accuracy of DSSR model include fully considering power flow, voltage constraints, and integration of DGs.

\section{Conflict of Interests}

The authors declare that there is no conflict of interests regarding the publication of this paper. None of the authors have a commercial interest, financial interest, and/or other relationship with manufacturers of pharmaceuticals,laboratory supplies, and/or medical devices or with commercialproviders of medically related services.

\section{Acknowledgments}

This work was supported by the National Natural Science Foundation of China (no. 51277129) and National High Technology Research and Development Program of China (863 Program) (no. 2011AA05A117).

\section{References}

[1] J. Xiao, W. Gu, C. Wang, and F. Li, "Distribution system security region: definition, model and security assessment," IET Generation, Transmission \& Distribution, vol. 6, no. 10, pp. 10291035, 2012.

[2] State Grid Corporation of China, Guidelines of Urban Power Network Planning, State Grid Corporation of China, Beijing, China, 2006 (Chinese).

[3] E. Lakervi and E. J. Holmes, Electricity Distribution Network Design, IET, Peregrinus, UK, 1995.

[4] K. N. Miu and H. D. Chiang, "Service restoration for unbalanced radial distribution systems with varying loads: solution algorithm," in Proceedings of the IEEE Power Engineering Society Summer Meeting, vol. 1, pp. 254-258, 1999.

[5] Y. X. Yu, "Security region of bulk power system," in Proceedings of the International Conference on Power System Technology (PowerCon '02), vol. 1, pp. 13-17, Kunming, China, October 2002.

[6] F. Wu and S. Kumagai, "Steady-state security regions of power systems," IEEE Transactions on Circuits and Systems, vol. 29, no. 11, pp. 703-711, 1982.

[7] Y. Yu, Y. Zeng, and F. Feng, "Differential topological characteristics of the DSR on injection space of electrical power system," Science in China E. Technological Sciences, vol. 45, no. 6, pp. 576584, 2002.

[8] S. J. Chen, Q. X. Chen, Q. Xia, and C. Q. Kang, "Steadystate security assessment method based on distance to security region boundaries," IET Generation, Transmission \& Distribution, vol. 7, no. 3, pp. 288-297, 2013.

[9] D. M. Staszesky, D. Craig, and C. Befus, "Advanced feeder automation is here," IEEE Power and Energy Magazine, vol. 3, no. 5, pp. 56-63, 2005.

[10] C. L. Smallwood and J. Wennermark, "Benefits of distribution automation," IEEE Industry Applications Magazine, vol. 16, no. 1, pp. 65-73, 2010.

[11] X. Mamo, S. Mallet, T. Coste, and S. Grenard, "Distribution automation: the cornerstone for smart grid development strategy," in Proceedings of the IEEE Power \& Energy Society General Meeting (PES '09), pp. 1-6, July 2009.

[12] P. W. Sauer, B. C. Lesieutre, and M. A. Pai, "Maximum loadability and voltage stability in power systems," International Journal of Electrical Power \& Energy Systems, vol. 15, no. 3, pp. 145-153, 1993.

[13] K. N. Miu and H. Chiang, "Electric distribution system load capability: problem formulation, solution algorithm, and numerical results," IEEE Transactions on Power Delivery, vol. 15, no. 1, pp. 436-442, 2000.

[14] F. Z. Luo, C. S. Wang, J. Xiao, and S. Ge, "Rapid evaluation method for power supply capability of urban distribution system based on $N-1$ contingency analysis of main-transformers," International Journal of Electrical Power and Energy Systems, vol. 32, no. 10, pp. 1063-1068, 2010. 
[15] J. Xiao, F. X. Li, W. Z. Gu, C. S. Wang, and P. Zhang, "Total supply capability and its extended indices for distribution systems: definition, model calculation and applications," IET Generation, Transmission and Distribution, vol. 5, no. 8, pp. 869-876, 2011.

[16] J. Xiao, X. X. Gong, and C. S. Wang, “Topology properties and algorithm of $\mathrm{N}-1$ security boundary for smart grid," Proceedings of the CSEE, vol. 34, no. 4, pp. 545-554, 2014 (Chinese).

[17] G. E. Shilov, An Introduction to the Theory of Linear Spaces, Dover, New York, NY. USA, 1975.

[18] J. R. Munkres, Topology, vol. 2, Prentice Hall, Upper Saddle River, NJ, USA, 2000. 


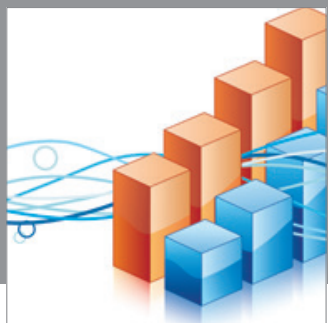

Advances in

Operations Research

mansans

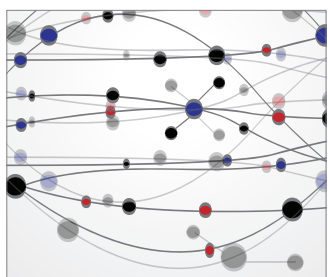

The Scientific World Journal
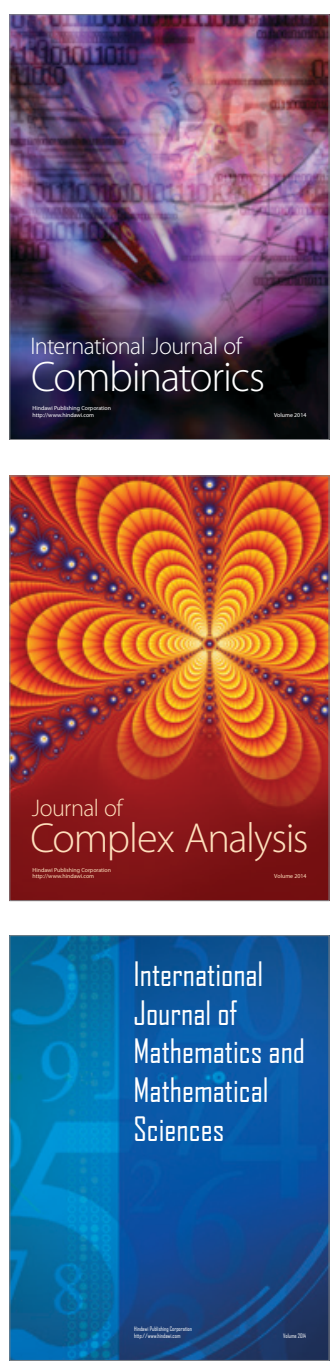
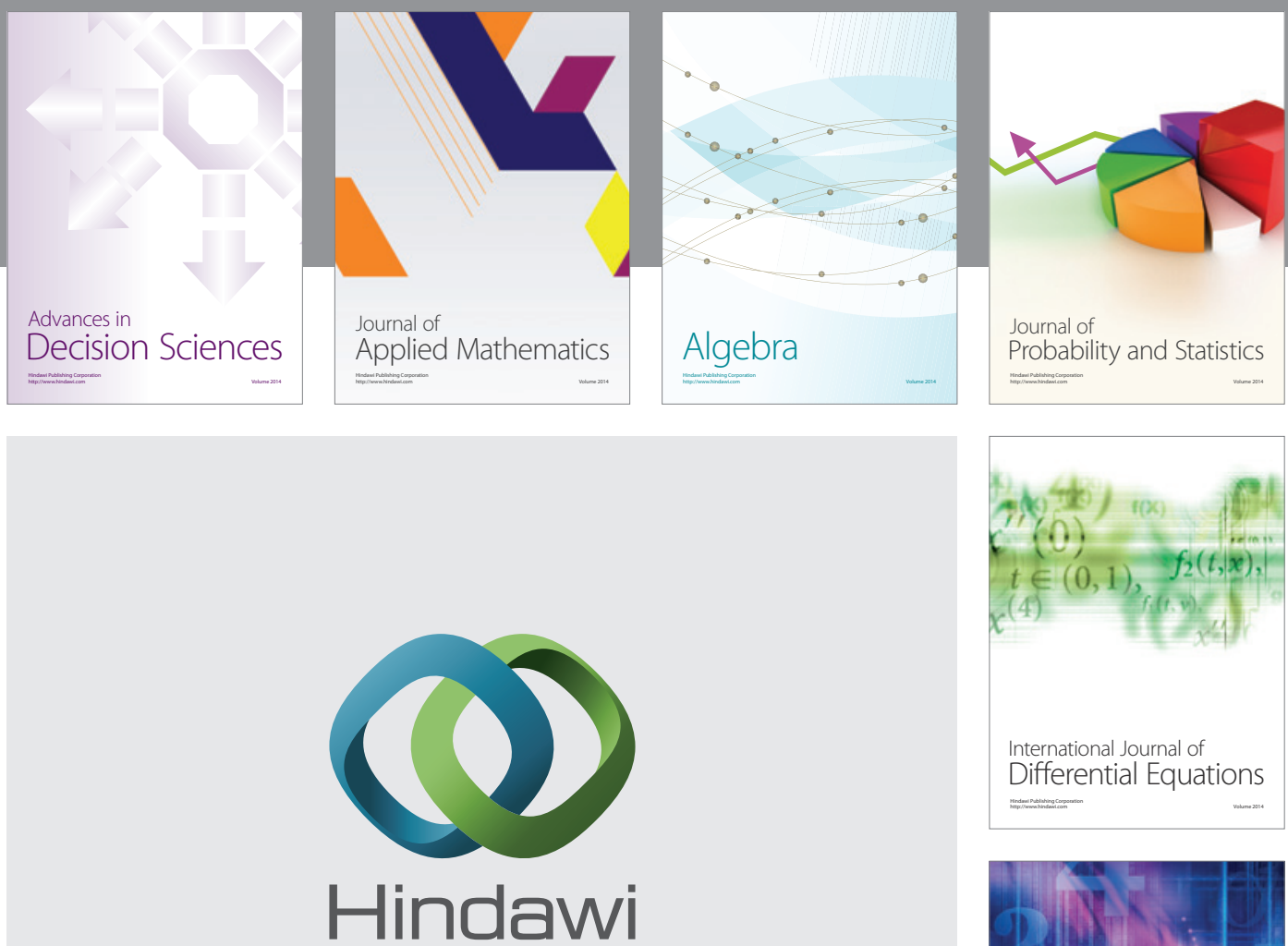

Submit your manuscripts at http://www.hindawi.com
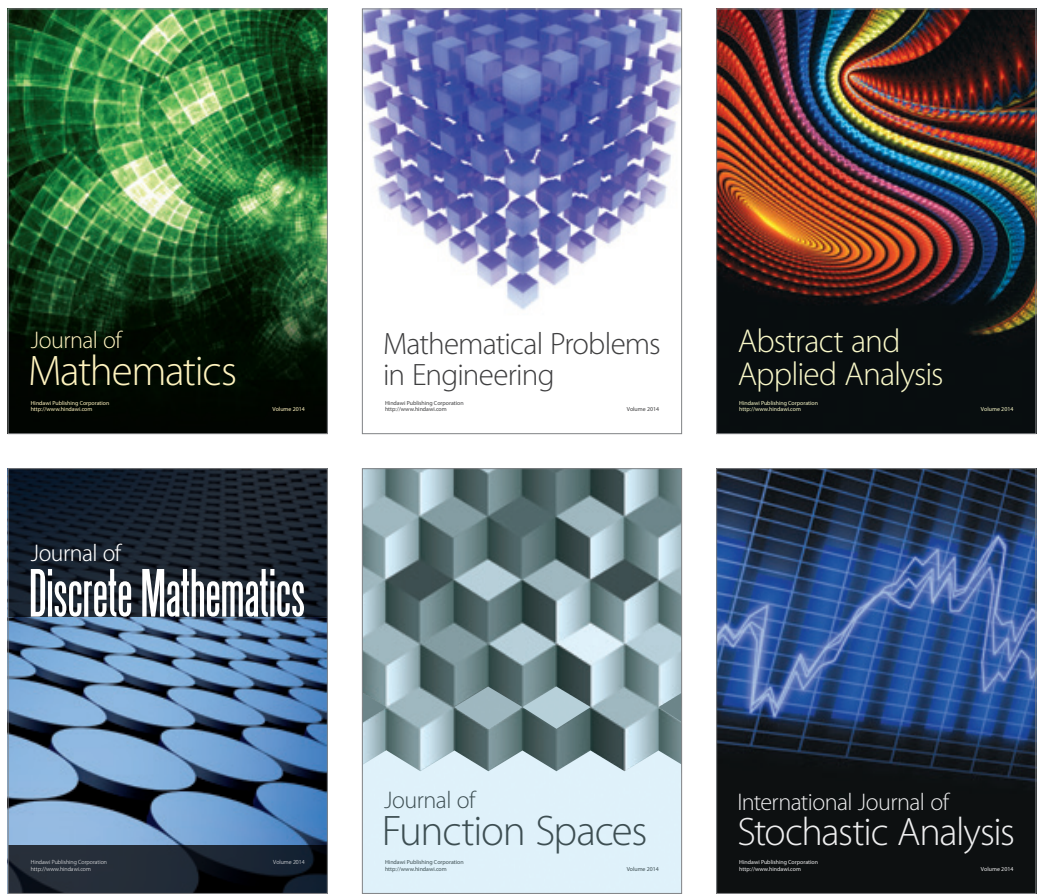

Journal of

Function Spaces

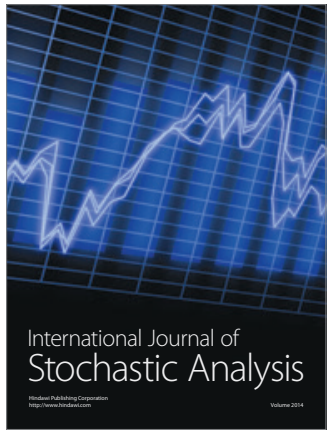

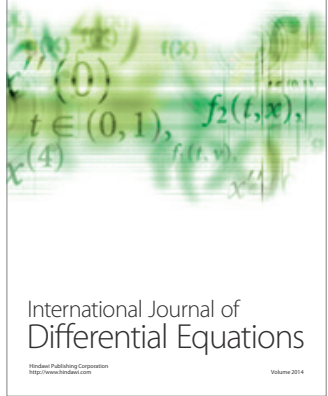
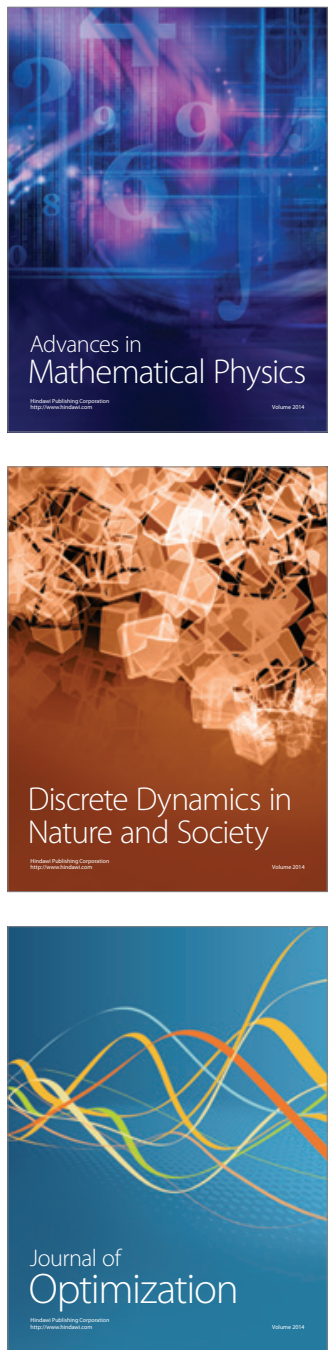